\title{
Diagnostic des Contraintes de Mise en Valeur Rizicole des Sols Fluvio-Marins du Marigot de Bignona, Basse Casamance, Sénégal
}

\author{
Ä̈dara C. A. Lamine Fall, \\ Yancouba Sane, \\ Laboratoire de Géomatique et d'Environnement (LGE) \\ Université Assane Seck de Ziguinchor (UASZ), Ziguinchor, Sénégal
}

\section{Résumé}

Situé en Basse Casamance, sud-ouest du Sénégal, le marigot de Bignona, a été durement éprouvé par la sécheresse des années 1970. La baisse pluviométrique et de l'écoulement fluvial y ont entraîné la salinisation et l'acidification des rizières. L'objectif de l'étude est d'analyser les contraintes de mise en valeur rizicole des sols fluvio-marins du marigot de Bignona pour la proposition de stratégies d'aménagement durables. Sept localités (Tenghory Transgambienne, Tenghory, Boutolate, Bindago, Piran, Koutenghor et Diourou) ont été choisies. Ce choix est motivé par l'ampleur de la dégradation des rizières par salinisation et acidification. La méthodologie comprend des enquêtes de terrain, l'analyse d'échantillons de sol et une cartographie de l'occupation du sol. Les résultats montrent une salinisation et une acidification généralisées des rizières dans le marigot de Bignona, avec une conductivité électrique comprise entre 0,01 et $9,77 \mathrm{mS} / \mathrm{cm}$ et un $\mathrm{pH}$ entre 4,7 à 7,8 . L'intrusion marine et l'oxydation de la pyrite sont, respectivement, les principaux facteurs responsables de la salinisation et l'acidification de ces sols. L'accumulation du fer en conditions anaérobies induit une autre contrainte, en l'occurrence la toxicité ferreuse. La combinaison de stratégies apparaît comme la meilleure méthode pour développer la riziculture dans le marigot de Bignona.

Mots clés : Salinisation, Acidification, Sols, Riziculture, Marigot de Bignona, Basse Casamance 


\title{
Diagnosis of Rice Growing Constraints in Fluvio- Marine Soils of the Bignona Valley, Lower Casamance, Senegal
}

\author{
Aidara C. A. Lamine Fall, \\ Yancouba Sane, \\ Laboratoire de Géomatique et d'Environnement (LGE) \\ Université Assane Seck de Ziguinchor (UASZ), Ziguinchor, Sénégal
}

\begin{abstract}
Located in Lower Casamance, southwestern Senegal, the Bignona valley was severely affected by the drought of the 1970s. The drop in rainfall and river flow brought about salinization and acidification of rice fields. The objective of this study is to analyze the constraints of rice growing in fluviomarine soils of the Bignona valley, in order to propose sustainable management strategies. Seven localities (Tenghory Transgambienne, Tenghory, Boutolate, Bindago, Piran, Koutenghor and Diourou) were selected. This choice is motivated by the important degradation of rice fields by salinization and acidification. The methodology includes field surveys, soil sample analysis and land cover mapping. The results show widespread salinization and acidification of rice-field soils, with soil electrical conductivity between 0.01 and $9.77 \mathrm{mS} / \mathrm{cm}$ and $\mathrm{pH}$ values between 4,7 to 7,8 . Marine intrusion and pyrite oxidation are, respectively, the main factors responsible for the salinization and acidification of these soils. The iron accumulation under anaerobic conditions induces another soil management constraint, namely the iron toxicity, which greatly affects the performance of rice growing. The combination of strategies appears as the most appropriate method to promote rice growing in the Bignona valley.
\end{abstract}

Keywords: Salinization, Acidification, Soils, Rice growing, Bignona valley, Low Casamance

\section{Introduction}

Région connue pour l'abondance de sa pluviométrie et la fertilité de ses sols, la Basse Casamance a été durement éprouvée par la période de sécheresse des années 1970. La baisse de la pluviométrie et de l'écoulement fluvial a eu pour conséquences dramatiques l'invasion des eaux marines dans tout le réseau hydrographique, la baisse généralisée du niveau des nappes, la 
salinisation et l'acidification des vasières occupées par la mangrove qui a disparu sur de grandes étendues. De nombreuses rizières, aussi bien salées (zones de mangrove) que douces (marigots inondés), ont été abandonnées (Albergel et al. 1992).

L'augmentation considérable des surfaces salées de 'tannes' au détriment de la mangrove à palétuviers en voie de dégradation et une progression du front de salinité vers les plateaux en sont actuellement les conséquences les plus visibles. Tous les marigots de la rive droite du fleuve Casamance, en particulier ceux de Baila et de Bignona sont confrontés à ce phénomène. La salinisation et l'acidification constituent ainsi les contraintes majeures de mise en valeur des terres du bassin fluvio-marin de Basse Casamance. Les moyens pour lutter contre elles existent, mais exigent tout d'abord de maîtriser convenablement la ressource eau, ce qui est loin d'être aisé dans une région si plate (Montoroi, 1993). Compte tenu de cette topographie locale plane, la sécheresse, en modifiant le régime hydrique, a provoqué une véritable catastrophe écologique à l'échelle régionale. Devant la persistance du déficit pluviométrique, l'excessive teneur en sels s'est étendue à la quasi-totalité des sols du bassin casamançais dont l'acidification s'est généralisée. Dans ce contexte spécifique, le rôle de l'acidité et de la très forte salinité n'est pas clairement établi, car elles subissent des variations importantes, fonction de la fréquence des marées et du rythme des saisons. L'équilibre écologique de la région a été modifié et certaines caractéristiques des sols ont été exacerbées (Diagne \& Cescas, 1997), malgré un retour pluviométrique enregistré en Afrique de l'Ouest depuis la fin du dernier millénaire (Descroix et al. 2015). L'objectif de ce travail est d'analyser les principales contraintes de mise en valeur rizicole des sols fluvio-marins du marigot de Bignona, Basse Casamance, sud-ouest du Sénégal, afin de proposer des stratégies d'aménagement efficaces et durables permettant de tirer profit de ces sols aux immenses potentialités. «Les sols des zones alluviales représentent un potentiel économique indéniable, en particulier pour l'obtention de rendements vivriers, notamment grâce à la riziculture, déjà largement développée en Basse-Casamance, mais qui pourrait être encore intensifiée par l'emploi de techniques d'aménagement adéquates» (Vieillefon, 1975). C'est pourquoi une étude détaillée des sols du domaine fluvio-marin de Basse Casamance est nécessaire, d'autant plus que les études pédologiques les plus récentes portant sur la région datent des années 1970-1990 (Aubrun \& Marius, 1986; Vieillefon, 1975 et 1977; Marius, 1985; Loyer et al. 1986; Posner et al. 1988; Montoroi, 1993, 1996a et 1996b; Boivin, 1991; Brunet, 1994...). Depuis lors, les données climatiques ont changé et beaucoup de paramètres relatifs à la salinité et l'acidité des sols ont évolué en plusieurs endroits du bassin de la Casamance (Diallo, 2016). La problématique de l'aménagement des sols du domaine fluvio-marin de Basse Casamance, établie 
sur des données anciennes, doit donc être révisée en tenant compte du contexte pédoclimatique actuel.

\section{Matériel et méthodes}

\subsection{Caractéristiques de la zone d'étude}

Le bassin du marigot de Bignona est compris entre les bassins des marigots de Diouloulou et Baila à l'Ouest et au Nord et du Soungrougrou à l'Est. A l'Ouest, la vaste dépression parcourue par le cours aval des marigots de Baila et Diouloulou correspond à l'ancien golfe Diouloulou-Tendouck occupé pendant les transgressions ouljienne et flandrienne. Au Nord, le marigot de Baila entaille le plateau sénégambien du Continental Terminal aux limites hydrogéologiques imprécises. A l'Est, la vallée du Soungrougrou est presqu'aussi large que la Casamance. Cette largeur démesurée au regard de la puissance du cours d'eau s'explique par l'ennoiement de la vallée de Bignona au cours de la dernière transgression. Ce comblement par des sables, vases et argiles de paléovallées profondes explique la configuration actuelle du réseau hydrographique en larges bas-fonds, ou estuaires s'ils sont envahis par les remontées marines. C'est aussi le paysage offert par le marigot de Bignona à une échelle plus modeste; à Bignona, le "lit mineur" a une vingtaine de mètres de large pour 1,5 $\mathrm{m}$ de profondeur (Vieillefon, 1975).

Le marigot de Bignona fait donc partie, avec ceux de Baila et de Diouloulou, des trois principaux marigots de rive droite du fleuve Casamance. C'est aussi le moins important. Son bassin versant couvre environ $800 \mathrm{~km}^{2}$ (encore que ses limites soient difficiles à préciser près du confluent avec la Casamance). Le cours principal du marigot à une longueur de $88 \mathrm{~km}$ dont 68 $\mathrm{km}$ sont soumis à l'influence des marées; c'est dire l'importance de la pénétration marine dans cette région (Olivry \& Chouret, 1981; Aubrun \& Marius, 1986).

\subsection{Méthodologie}

Cette étude a porté sur 7 localités situées le long du marigot de Bignona, en l'occurrence Tenghory Transgambienne, Tenghory, Boutolate, Bindago, Piran, Koutenghor et Diourou (figure 1). Ce choix est motivé d'une part par l'importance de l'activité rizicole, et d'autre part par l'ampleur de la dégradation des rizières par salinisation et acidification dans ces localités.

Dans chacune des localités, la dégradation des rizières a été étudiée à travers ses causes (facteurs responsables), conséquences (impacts), et les méthodes de lutte ont été évaluées. La méthodologie se subdivise en trois rubriques: des analyses d'échantillons de sol représentatifs, une cartographie diachronique de l'occupation du sol qui a permis d'avoir une idée de la dynamique de la salinisation et de l'acidification des sols dans la zone d'étude et des enquêtes de terrain. 


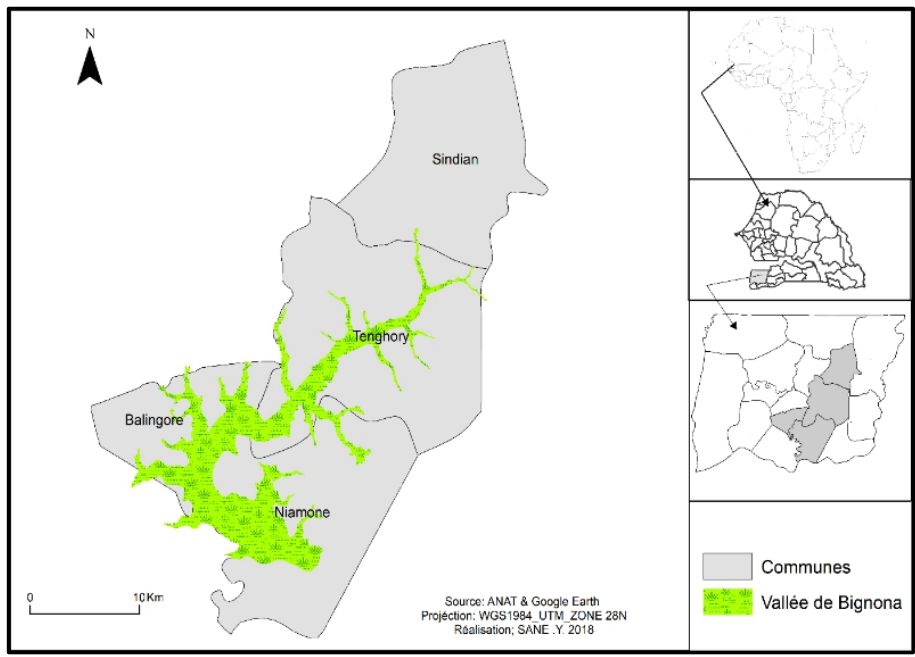

Figure 1 - Carte de localisation de la vallée de Bignona

Pour l'analyse des paramètres physico-chimiques des sols en rapport avec la salinisation et l'acidification (conductivité électrique, CE; et potentiel Hydrogène, $\mathrm{pH}$ ), 18 échantillons dans 9 profils de $50 \mathrm{~cm}$ de profondeur ont été prélevés. Ainsi, 2 échantillons de sol ont été prélevés dans chaque profil, à deux profondeurs différentes: $0-25 \mathrm{~cm}$ et $25-50 \mathrm{~cm}$. Les analyses ont été effectuées au laboratoire du département de Physique de l'Université Assane Seck de Ziguinchor et nous avons adopté un ratio de 1/5 pour la $\mathrm{CE}$ et un ratio de $1 / 2,5$ pour le $\mathrm{pH}$.

Des analyses diffractométriques (DRX) complémentaires ont été effectuées au laboratoire de l'Institut d'écologie et des sciences de l'environnement de Paris (iEES Paris, Centre IRD France-Nord), sur quelques échantillons de sols pour apprécier leur composition chimique et minéralogique.

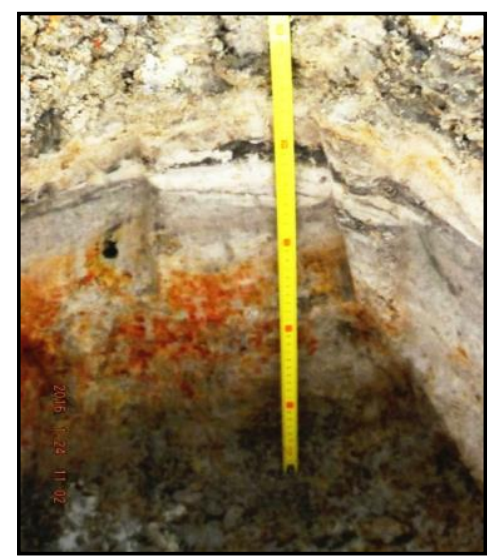

Photo 1 - Profil de sol dans la vallée de Bignona mettant en exergue les réactions d'oxydoréduction du fer 
La cartographie de l'occupation du sol a été réalisée à partir des données géospatiales et des travaux de terrain effectués en 2015 et en 2016. Les données géospatiales concernent des images satellitaires (corona 1968), des captures de Google Earth (janvier 2006 et janvier 2015) et des points GPS pour la localisation des données recueillies sur le terrain et la confirmation ou l'infirmation des classes des différentes unités de paysage cartographiées. Ces données ont été analysées par une approche photo-interprétation. Ainsi, une série de géoréférencement et de numérisation des contours des unités du paysage a été effectuée. Ce qui nous a permis de faire une analyse diachronique de la dynamique de l'occupation du sol de 1968 à 2015.

Les enquêtes de ménages ont été réalisées à l'aide d'un questionnaire administré aux concessions qui s'activent dans la riziculture, soit 16 concessions par village. Le choix de 16 concessions se justifie par un souci d'harmonisation, car la localité de Bindago compte au total 16 concessions. Ce qui a permis d'interroger $7 \times 16$ concessions, soit 112 concessions. Ensuite, l'échantillonnage par grappe a été adopté. Il a consisté à interroger tous les ménages qui s'activent dans la riziculture dans chaque concession. L'unité d'échantillonnage est la concession et l'unité de référence est le chef de ménage riziculteur.

\section{Résultats et discussion}

\subsection{La salinisation des sols dans le marigot de Bignona}

Les résultats obtenus révèlent une variation horizontale et verticale de la salinité des sols dans le marigot de Bignona. Elle se fait, d'une part à travers l'inondation des sols par les eaux salées du réseau hydrographique qui envahissent ces zones basses à l'occasion des grandes marées (processus horizontal) et, d'autre part, par remontée capillaire de la nappe salée dans les profils en saison sèche (processus vertical).

Le processus horizontal de salinisation parait le plus important dans le marigot de Bignona. Il entraine l'augmentation de la salinité des sols et des nappes et explique la forte salinité des sols dans les localités situés à proximité du lit du marigot comme Diourou (P1 et P2 avec 5,45 mS/cm et 9,5 mS/cm, respectivement) et Koutenghor (P1 et P2 avec des valeurs respectives de 9,77 $\mathrm{mS} / \mathrm{cm}$ et $6,25 \mathrm{mS} / \mathrm{cm}$ ). En effet, les inondations successives se traduisent par des apports de sels qui cristallisent sous l'effet de l'évaporation à la surface du sol en formant des couches épaisses à structure poudreuse de type "moquette" (Marius, 1985; Sadio, 1991).

Par rapport aux horizons, nous constatons globalement que pour chaque profil, l'horizon de surface $(0-25 \mathrm{~cm})$ est plus affecté par le sel que l'horizon inférieur $(25-50 \mathrm{~cm})$. Ainsi, dans l'ensemble, les valeurs de la CE varient en moyenne entre 0,03 et $9,77 \mathrm{mS} / \mathrm{cm}$ pour le premier horizon $(0-25$ $\mathrm{cm})$, et entre 0,01 et $2,21 \mathrm{mS} / \mathrm{cm}$ pour le second horizon $(25-50 \mathrm{~cm})$. 
Cette variation verticale de la salinité matérialisée par une augmentation de la salinité des sols en surface, reste liée à la dynamique de la nappe salée. En effet, pendant toute la saison sèche, les fortes températures $\left(25\right.$ à $\left.40^{\circ} \mathrm{C}\right)$ qui règnent sur le site entrainent un important flux ascendant d'eaux salées de la nappe et une concentration des sels en surface. La présence de croûtes de sels en surface visibles sur certains sites, en particulier à Diourou, Koutenghor, en est une parfaite illustration.

\subsection{L'acidification des sols et la toxicité ferreuse}

Aucun gradient latéral de distribution de l'acidité n'a été observé. En effet, les valeurs du pH obtenues sont marquées par leur importante variabilité. Ce qui reflète une certaine hétérogénéité spatiale des propriétés édaphiques des sols dans le marigot de Bignona. La baisse des valeurs du $\mathrm{pH}$ dans 1'horizon $25-50 \mathrm{~cm}$ a toutefois été constatée dans tous les profils étudiés, excepté pour Tenghory et Tenghory Transgambienne; ce qui traduit une augmentation de l'acidité en profondeur (tableau 1). La présence de croûtes salées en surface pourrait expliquer une telle tendance, avec la forte présence d'ions $\mathrm{Na}^{+}$qui neutralisent les effets de l'ion $\mathrm{H}^{+}$responsable de l'acidité de ces sols.

Les valeurs du $\mathrm{pH}$ obtenues dans tous les profils $(4,7<\mathrm{pH}<7,8)$ confirment ainsi l'acidité des sols dans le marigot de Bignona.

Tableau 1: CE et pH du sol dans les différentes localités de la zone d'étude (source: Sané,6)

\begin{tabular}{|l|c|c|c|c|}
\hline Localité & \multicolumn{2}{|c|}{ Horizon (cm) } & \multicolumn{2}{c|}{ Horizon (cm) } \\
\hline & $\mathbf{0 - 2 5}$ & $\mathbf{2 5 - 5 0}$ & $\mathbf{0 - 2 5}$ & $\mathbf{2 5 - 5 0}$ \\
\hline & \multicolumn{2}{|c|}{$\mathrm{pH}$} & \multicolumn{2}{c|}{ CE $(\mathrm{mS} / \mathrm{cm})$} \\
\hline Tenghory Tr. & 7,0 & 7,1 & 0,03 & 0,02 \\
\hline Tenghory & 7,7 & 7,8 & 0,09 & 0,01 \\
\hline Boutolate & 6,8 & 6,5 & 5,83 & 2 \\
\hline Bindago & 7,9 & 6,9 & 0,2 & 0,08 \\
\hline Piran & 5,4 & 4,9 & 1,14 & 1,03 \\
\hline Koutenghor P1 & 6,9 & 6,2 & 9,77 & 2,21 \\
\hline Koutenghor P2 & 5,2 & 5,0 & 6,25 & 1,69 \\
\hline Diourou P1 & 5,1 & 4,7 & 5,45 & 0,57 \\
\hline Diourou P2 & 6,7 & 6,1 & 9,5 & 1,25 \\
\hline
\end{tabular}


L'acidification, dans cette partie de la Basse Casamance, résulte de l'oxydation de la pyrite $\left(\mathrm{FeS}_{2}\right)$ présente dans les sédiments fluvio-marins de toute la côte ouest du Sénégal (Vieillefon, 1977; Marius, 1985; Loyer et al. 1986; Sadio, 1991; Boivin, 1991; Montoroi, 1993; Montoroi et al, 1993; Montoroi, 1996a et 1996b; Brunet, 1994; Fall et al. 2014). En effet, l'hydromorphie permanente, qui prévalait dans ces sols avant la sécheresse des années 1970, avait créé des conditions anaérobies permettant la réduction des sulfates marins en sulfures avec, notamment, la formation d'un sulfure de fer: la pyrite $\left(\mathrm{FeS}_{2}\right)$. L'abaissement des nappes de bas-fonds a provoqué une acidification par oxydation de ces composés sulfurés sous l'action de bactéries sulfato-oxydantes (en particulier le thiobacillus ferrooxidans). La pyrite s'est ainsi transformée en un sulfate de fer caractéristique : la jarosite $\left[\left(\mathrm{KFe}^{3}\left(\mathrm{SO}_{4}\right)_{2}(\mathrm{OH})_{6}\right]\right.$ avec production d'acide sulfurique $\left(\mathrm{H}_{2} \mathrm{SO}_{4}\right)$. Le $\mathrm{pH}$ in situ a chuté à des valeurs souvent inférieures à 5 et de vastes zones de sols potentiellement sulfatés acides (mangroves, bas-fonds) se sont transformées en sols sulfatés acides (tannes).

A cette acidité, qui est devenue une sérieuse contrainte de mise en valeur rizicole, est venue s'ajouter une toxicité ferreuse, contribuant à accentuer davantage la dégradation des rizières dans le marigot de Bignona. En effet, l'acidité résultant de l'oxydation de la pyrite a occasionné l'enrichissement des sols sulfatés acides des bas-fonds en fer. Ce fer peut provenir de la mise en solution in situ dans le bas-fond ou par lessivage et ruissellement des pentes adjacentes du bas-fond (Audebert, 2006). Le fer est en grande partie sous forme de fer ferrique $\mathrm{Fe}^{3+}$ dans les sols de plateau et sur les pentes. Dans ces situations topographiques, il est sous forme insoluble. Le fer sous cette forme est très peu absorbé par le riz et des cas de carence en fer ont pu être notés sur les sols rouges ferrugineux tropicaux lessivés du plateau de Djibélor, en Casamance. Les conditions d'hydromorphie, favorisent la réduction du fer ferrique $\mathrm{Fe}^{3+}$ en fer ferreux $\mathrm{Fe}^{2+}$ (Khouma M, 2006). La toxicité ferreuse pénalise fortement les performances de la riziculture traditionnelle en Basse et Moyenne Casamance.

Les analyses diffractométriques effectuées ont permis de confirmer les résultats des analyses physico-chimiques (figure 2). Les principaux minéraux présents dans ces sols sont, par ordre d'importance : quartz $(\mathrm{Q})$, kaolinite (Ka), halite $(\mathrm{NaCl})$. La présence de ce dernier minéral justifie le caractère salé des sols étudiés, notamment ceux de Diourou et Koutenghor. Aussi, la proximité de ces localités avec le cours d'eau confirme-t-elle l'origine marine de la salinisation des rizières dans le marigot de Bignona. 


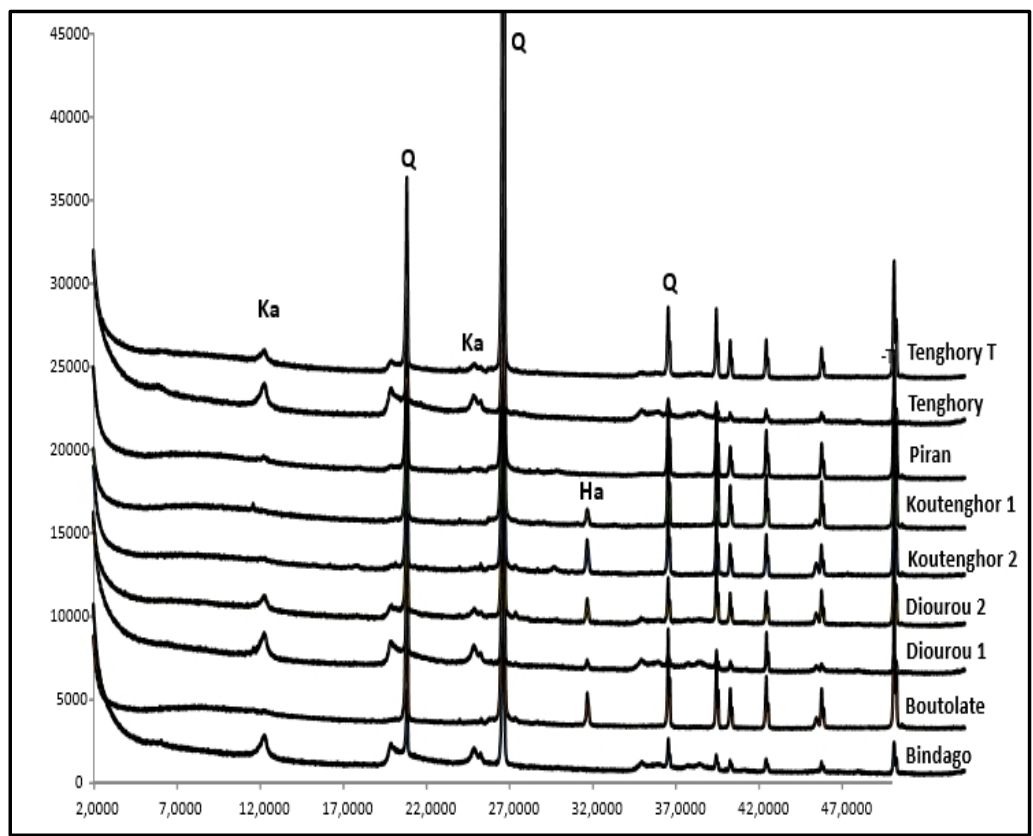

Figure 2 : Principaux minéraux présents dans les sols de la vallée de Bignona

\subsection{La dynamique des unités paysagères}

La figure 3 montre l'occupation du sol dans la zone d'étude, entre 1968 et 2015. La tendance générale qui se dégage de cette figure reste la progression de la superficie des rizières abandonnées aux dépens des rizières en activité et de la végétation de terre ferme. La sécheresse des années 1970 est à l'origine de cette tendance, avec la salinisation et l'acidification des rizières et leur abandon progressif par les populations locales.

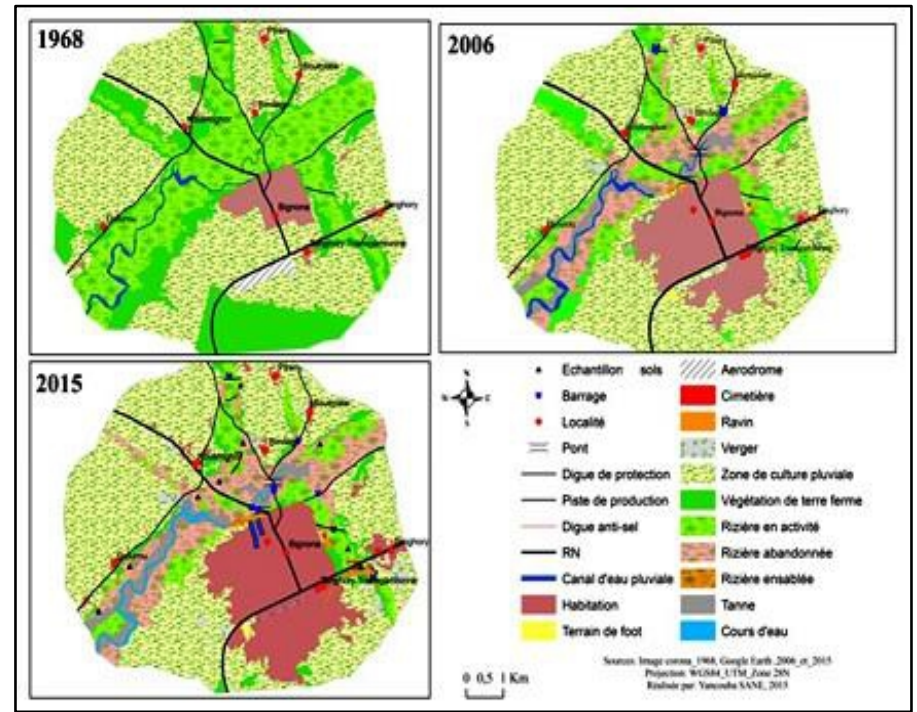

Figure 3 : Carte d'occupation du sol dans la zone d'étude, entre 1968 et 2015 
L'interprétation de la carte de l'occupation du sol de 1968 permet de voir que les rizières étaient bien présentes dans le marigot de Bignona à cette période. Par contre, depuis les années de sécheresse de 1970 (cartes de 2006 et 2015), cette unité du paysage connaît des modifications induites en particulier par la baisse de la pluviométrie, qui a entraîné la salinisation et l'acidification des sols. Ces phénomènes ont entraîné une forte dégradation des rizières qui se traduit au niveau des cartes par l'abandon progressif de certaines parcelles rizicoles. Ainsi, entre 1968 et 2015, près de la moitié de la superficie des rizières en activité est abandonnée. Il en est de même de la végétation de terre ferme qui a souffert du déficit pluviométrique, mais aussi de l'action humaine à travers une déforestation massive et la progression du bâti qui a empiété sur d'anciennes zones rizicoles et de culture pluviale (figure $3)$.

L'autre constat qui découle de l'interprétation des cartes d'occupation du sol reste l'apparition des surfaces de «tannes» (vastes étendues de terres salées et incultes qui se développent généralement en arrière-plan de la mangrove). Avant les années de sécheresse de 1970, les tannes n'existaient pas dans la zone d'étude. Ce que confirment les populations enquêtées qui n'avaient pas noté l'existence de cette unité paysagère dans leurs localités. Cependant, pendant et après la sécheresse, certaines rizières, sous l'effet de la salinisation et de l'acidification, sont devenues des tannes. Cela est particulièrement visible sur les cartes d'occupation du sol de 2006 et 2015 où on voit l'apparition et l'extension progressive des surfaces de tannes au détriment des surfaces rizicoles.

\subsection{Propositions de stratégies durables de mise en valeur des terres rizicoles du marigot de Bignona}

La Casamance fait partie de l'ensemble géographique ouest-africain des rivières du Sud (Dresch, 1949) qui constitue l'une des aires de riziculture africaine dont l'histoire est très ancienne (Portères, 1950). Cette riziculture a donné lieu à des aménagements hydro-agricoles spectaculaires, les paysans casamançais étant réputés pour leurs capacités d'aménagement qui autorisent la pratique de la riziculture dans un milieu soumis notamment à la salinité (Pélissier, 1966 ; Linares, 1992). La Casamance peut également se prévaloir d'une expérience dans la durée en matière d'interventions publiques ou privées qui se sont attachées à promouvoir des solutions techniques pour enrayer les processus de dégradation affectant la riziculture, les vallées et plus globalement l'écosystème cultivé dans son ensemble. Cependant, le bilan de ces interventions est loin d'être positif: on ne dénombre guère de succès des interventions extérieures sur ces problématiques, malgré des diagnostics scientifiques et des recherches pertinentes, tant sur les mécanismes de dégradation du milieu que sur les solutions techniques à mettre en œuvre 
(Bosc, 2005). Pourquoi un tel bilan? Comment expliquer l'échec de la presque totalité des stratégies d'aménagement des rizières de Basse Casamance? Comment tirer profit des immenses potentialités rizicoles de cette région, la plus pluvieuse du Sénégal?

La présente recherche a démontré la dégradation continue des terres rizicoles du marigot de Bignona par salinisation et acidification. Devant l'ampleur du phénomène et l'inefficacité des stratégies de lutte appliquées séparément, la mise en valeur durable de ces terres ne pourra être obtenue que sur la durée par une combinaison de techniques mécaniques, organiques, minérales et agronomiques.

\subsubsection{La lutte mécanique : digues anti-sel et micro-barrages}

Pour lutter contre la salinisation qui a considérablement réduit les superficies rizicoles de Basse Casamance, des aménagements hydroagricoles ont été mis en place dans les années 1980-1985 (Brunet et al. 1994). Deux types d'aménagements ont ainsi vu le jour pour combattre les effets de la salinité. D'une part, des grands projets de barrages anti-sel (Affiniam et Guidel) ont été construits, d'autre part, à l'instar des populations locales et des organismes de développement, de petits ouvrages anti-sel ont été édifiés pour arrêter l'entrée des eaux salées à l'intérieur des petites vallées alluviales (Albergel et al. 1992). Aujourd'hui, force est de constater que malgré tous les aménagements réalisés, la salinisation et l'acidification des terres rizicoles s'intensifient dans toute la Basse Casamance et progressent de façon insidieuse dans le temps et dans l'espace, affectant même les zones de terrasses et de plateau jusque-là épargnées.

Face à ce problème, les riziculteurs de Basse Casamance en général et du marigot de Bignona en particulier, ont développé des stratégies pour préserver leur outil de production. L'abondance des précipitations permet en effet de maintenir un équilibre propice à cette céréale, sur des terres naturellement salées par des intrusions marines dans les nappes souterraines. Cette précaire stabilité est toutefois rompue par la sécheresse persistante dans le pays depuis plusieurs décennies (Montoroi et Fall, 2017). La concentration de sel dans les sols, insuffisamment lessivés par les pluies, augmente au point d'affecter les rendements ou de compromettre la production.

Pour lutter contre cette lente et inexorable dégradation de leurs terres, ils ont mis en place des outils spécifiques. Côté mer et mangrove, ils ont bâti des digues pour empêcher la progression de la langue salée. Côté terre, ils ont édifié des petits barrages et des réservoirs, vers lesquels sont canalisées les eaux des pluies (photo 2). La ressource ainsi précieusement thésaurisée peut être entièrement mobilisée pour créer une lame d'eau douce dans les rizières. Grâce à ces efforts, déployés à l'échelle de la parcelle ou du village, certaines communautés sont parvenues à maintenir leur activité. Ces infrastructures sont 
néanmoins difficiles à maintenir en état, il faut sans cesse les consolider, les réparer ou les reconstruire. Une autre limite des aménagements mécaniques reste leur efficacité partielle. En effet, les micro-barrages permettent simplement de stopper l'écoulement de surface, mais n'agissent pas sur l'écoulement souterrain. Quant aux digues anti-sel, ils souffrent de l'absence d'un dispositif de drainage adapté permettant d'évacuer les eaux chargées de sel après lessivage. Ainsi, toute stratégie mécanique d'aménagement des terres rizicoles du marigot de Bignona devra au préalable répondre aux questions suivantes: comment canaliser l'eau de pluie pour lessiver les sels et favoriser le drainage des eaux salées? Comment drainer les couches superficielles et maintenir l'hydromorphie des couches profondes afin d'éviter l'oxydation de la pyrite et l'acidification des sols?
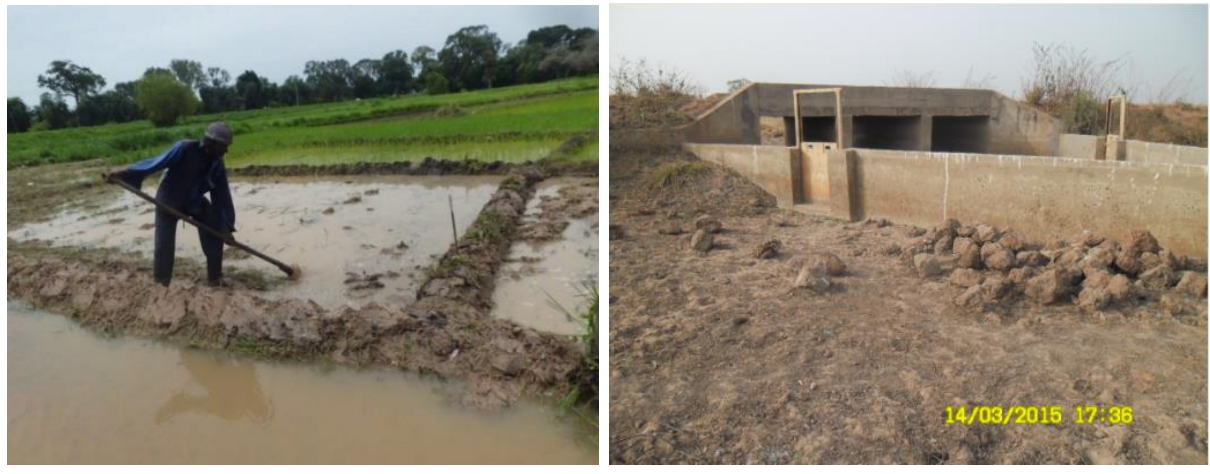

Photo 2 : Quelques méthodes de lutte mécanique dans le marigot de Bignona: endiguement et micro-barrage (Source : Sané, 2015)

Le problème principal du drainage demeure la définition d'un calendrier de travail pour les activités à mener. Une erreur d'appréciation peut nuire à l'efficacité des aménagements. Il faut donc choisir le bon moment pour évacuer les eaux chargées de sel en ouvrant les vannes: une ouverture tardive entraîne la resalinisation du profil après évaporation de l'eau et une ouverture précoce ne permet pas un lessivage conséquent des sels. Une fois que les terres sont préservées des principaux facteurs de dégradation, des mesures d'accompagnement, ou stratégies de lutte complémentaires doivent être entreprises pour une meilleure expression de leur fonction de production dans la durabilité.

\subsubsection{La lutte organique}

L'épandage de fumier, compost, coques d'arachide, résidus de cultures, sciure de bois, ou écailles de poisson à l'approche de l'hivernage, est une technique pratiquée par la majorité des paysans du bassin du Sine-Saloum (centre-ouest du Sénégal). Le principe de base est simple: lutter contre la salinisation des sols, c'est favoriser le lessivage des sels, le remplacement de 
l'ion sodium $\left(\mathrm{Na}^{+}\right)$par un autre cation (le $\mathrm{Ca}^{2+}$ par exemple) qui modère le milieu. Cette technique s'est révélée très efficace dans la récupération des terres salées. En effet, la matière organique favorise le lessivage des sels à travers les acides organiques qui fixent les ions sodium et les entraînent avec les eaux de pluie (N'gom, 1999). L'expérience du PROGERT (Projet de gestion et de restauration des terres dégradées du Bassin arachidier) en 2006 sur les tannes enherbées de Koutal (région de Kaolack, bassin du SineSaloum) en est une parfaite illustration. Cette étude a révélé que la coque d'arachide est riche en ions calcium. A une dose de deux (02) à quatre (04) tonnes/ha sur sol argileux et de huit (08) à dix (10) tonnes/ha sur sol sableux, la coque d'arachide contribue de façon efficiente à la réorganisation de la structure du sol et à l'amélioration de sa fertilité.

\subsubsection{La lutte minérale}

L'utilisation d'amendement du phosphogypse (PG) comme intrant, un produit dérivé des phosphates naturels $(\mathrm{PN})$, est une pratique anti-saline efficace dans les vallées rizicoles. Ce produit, appliqué dans des champsécoles de producteurs en moyenne Casamance, sous la supervision de l'INP, à raison de $1 \mathrm{~T} / \mathrm{ha}$, a augmenté de plus de 50\% les rendements de riz (INP \& Assolucer, 2009). Mais la particularité des terres rizicoles de Basse Casamance c'est également leur caractère acide dû à la présence de la pyrite. Récupérer ces terres salées et sulfatées acides suppose donc lutter contre l'acidité. Les procédés de lutte minérale s'avèrent de ce fait indispensables. Pour ce faire, la mise en valeur de ces sols devra s'attaquer tout d'abord à l'abaissement de l'acidité par la correction du $\mathrm{pH}$ à l'aide des techniques de chaulage ou des apports de débris de phosphates (Sadio, 1991). Le chaulage est indiqué par de nombreux auteurs (Bèye, 1973 ; Khouma et al. 1982; Dent, $1986 . .$. entre autres) comme la principale méthode de correction du $\mathrm{pH}$ des sols sulfatés acides. Grâce à l'apport du calcium $\left(\mathrm{Ca}^{2+}\right)$, l'acidité sera progressivement neutralisée; ce qui permettra le relèvement du $\mathrm{pH}$. L'utilisation des débris de phosphates par Boivin et al. (1987) en Casamance, a permis d'améliorer le pH d'un sol sulfaté acide salé qui est passé de 3,5 à 5,0 et d'assurer la production du riz. L'application de cette méthode se fera d'abord à petite échelle, pour en évaluer le coût et l'impact, avant sa large diffusion.

\subsubsection{La lutte agronomique ou culturale}

Il s'agit de limiter les pertes d'eau par remontée capillaire et évaporation. Pour ce faire, le travail du sol en surface et sa couverture par une couche de paille s'avèrent efficaces. 


\section{Le labour superficiel du sol}

L'étude des états de surface a permis de mieux comprendre le mode de fonctionnement de la composante superficielle des sols sulfatés acides salés du marigot de Bignona. Elle a en particulier révélé les contraintes majeures qui se posent au site et qui constituent un obstacle aux stratégies de récupération.

Une de ces contraintes demeure la cristallisation du sel en surface au niveau des zones dénudées, notamment dans les localités de Diourou et Koutenghor. Ce phénomène résulte des remontées capillaires d'eau salée à partir de la nappe, favorisées par l'intense évaporation pendant une longue période de l'année (mars à juin). Il a été démontré que la récupération de ces sols sulfatés acides salés devra d'abord s'attaquer à l'influence de l'eau salée provenant de l'estuaire (marées hautes) ou de la nappe. L'endiguement permet donc de régler simplement une partie du problème en réduisant l'invasion marine (composante horizontale). Pour ce qui est de la nappe salée (composante verticale), la solution pourrait partiellement résider dans le labour superficiel du sol. Ce travail du sol crée une rupture dans la remontée de l'eau salée. La zone de discontinuité ainsi créée entre l'horizon labouré et l'horizon sous-jacent constitue un tampon, sorte de barrière empêchant l'eau chargée de sel d'accéder à la surface du sol. L'impact du travail de la couche superficielle du sol a été expérimenté par Sadio (1991) dans le bassin du SineSaloum pour démontrer la rupture de capillarité provoquée. Il a pu ainsi constater une diminution de $30 \%$ de la salinité dans la zone labourée par rapport à celle non travaillée.

Cette expérience pourrait être reproduite dans le marigot de Bignona, compte tenu des similitudes hydro-géomorphologiques (domaine fluviomarin) et édaphiques (sols sulfatés acides salés, présence de mangroves et de tannes) entre ces deux zones. La pluviométrie nettement plus importante en Basse Casamance constitue un réel avantage.

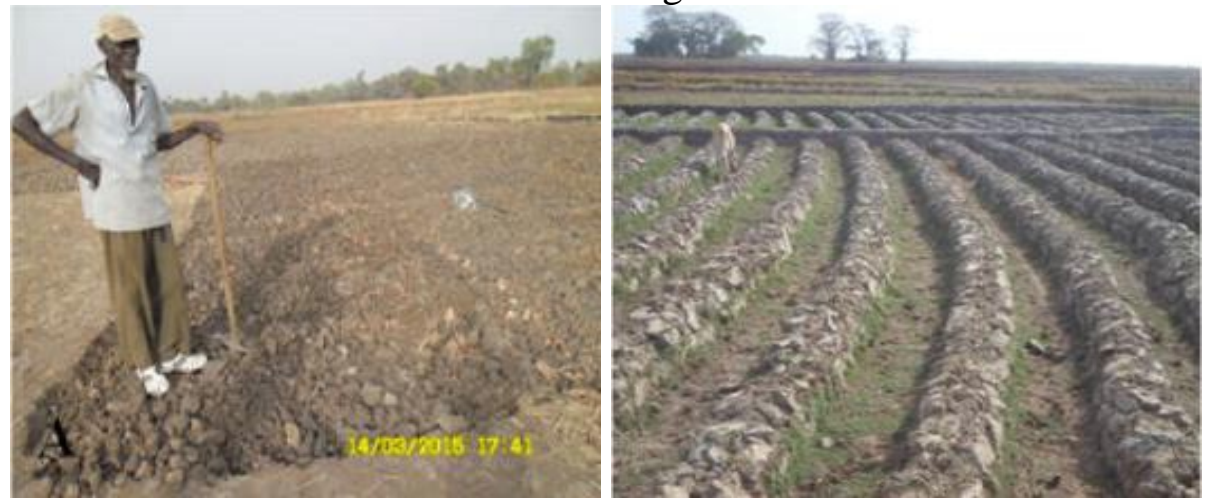

Photo 3 : Labour superficiel du sol dans le marigot de Bignona (Source : Sané, 2015) 


\section{Le Paillage (ou «mulching»)}

Dans l'optique d'une diminution des pertes d'eau par évaporation, la couverture de la surface du sol par une couche de paille pourrait être une solution. Par la même occasion, l'humidité du sol superficiel sera préservée, ce qui constitue un obstacle aux phénomènes d'encroûtement ou de cristallisation. Protéger la surface du sol par une couverture morte (paille) empêchera ainsi la resalinisation du profil déjà lessivé. L'effet du paillage sur le dessalement a été efficacement expérimenté par Bèye (1973) en Casamance. Il a pu constater une réduction de $50 \%$ de la salinité dans la partie supérieure du profil au bout de 4 ans malgré des années peu pluvieuses. Ce qui a permis la riziculture sur ces sols avec un rendement nettement plus élevé que celui des terres non aménagées.

La reprise pluviométrique constatée ces dernières années en Basse Casamance et qui annonce la fin probable du cycle de sécheresse devrait faciliter une telle pratique. D'autant plus que le lessivage du sel pendant l'hivernage s'accompagne d'un développement important du tapis herbacé. Cette herbe qui est exploitée par les riziculteurs pour nourrir leur bétail ou renouveler les toitures de leurs maisons pourrait être fauchée et conservée sur le site. Son dépôt à la surface du sol des rizières permettra d'y conserver l'humidité pendant une bonne partie de la saison sèche.

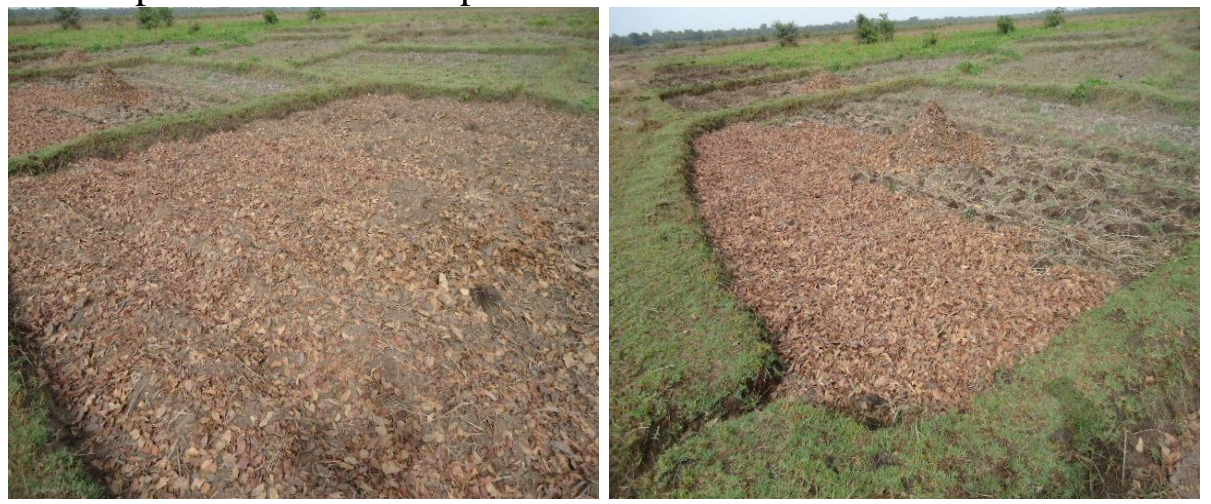

Photo 4 : Quelques techniques de «mulching» à Siganar, en Basse Casamance (Source :

Sané, 2015)

\section{Conclusion}

Au terme de cette étude, on peut retenir que les terres rizicoles du marigot de Bignona, plus particulièrement dans les villages de notre zone d'étude (Tenghory Transgambienne, Tenghory, Boutolate, Piran, Bindago, Koutenghor et Diourou) ont connu une dégradation pendant et après les années de sécheresse, à l'instar de toute la Basse Casamance. Cette dégradation qui se manifeste par la salinisation et l'acidification des sols a des conséquences environnementales et socio-économiques. L'abandon de 
plusieurs parcelles affectées est observé dans toutes les rizières des localités situées dans le marigot.

L'étude a démontré l'existence de différentes méthodes de lutte qui pourraient permettre, sinon de stopper, du moins réduire la dégradation des rizières du marigot de Bignona par salinisation et acidification. Toutefois, force est de constater qu'aucune stratégie isolée ne présente une efficacité réelle. Ainsi, à défaut d'une reprise pluviométrique conséquente, la solution pourrait résider dans la combinaison des stratégies.

\section{References:}

1. Albergel, J., Brunet, D., Dubée, G., Montoroi, J-P., \& Zante, P. (1992). Gestion d'un barrage anti-sel en basse Casamance (Sénégal). In: Carré Paul (Ed.), Usage agricole de l'eau. Paris: ORSTOM, (pp. 33-45), (Colloques et Séminaires). Journées Hydrologiques de l'ORSTOM, 6. Montpellier (FRA).

2. Aubrun, A. \& Marius, C. (1986). Etude pédologique sur la vallée de Bignona en Casamance. République du Sénégal, ministère de l'hydraulique; direction des aménagements et des infrastructures hydro-agricoles ; Rapport définitif; 113 p + annexes.

3. Audebert, A., Narteh, LT., Kiepe, P., Millar, D., \& Beks, B. (2006). Toxicité ferreuse dans les systèmes à base riz d'Afrique de l'ouest. Rapport ADRAO (Centre du Riz pour l'Afrique), 198 p.

4. Bèye, G. (1973). Une méthode simple de dessalement des sols des tannes de Casamance: le paillage. Agronomie Tropicale. $\mathrm{n}^{\circ} 28$, pp. 537-548.

5. Boivin, P. (1991). Caractérisation physique des sols sulfatés acides de la vallée de Katouré (basse Casamance, Sénégal): étude de la variabilité spatiale et relation avec les caractéristiques pédologiques. Paris: ORSTOM, 231 p. (Etudes et Thèses).

6. Bosc, P-M. (2005). A la croisée des pouvoirs: une organisation paysanne face à la gestion des ressources, Basse Casamance, Sénégal. Paris: IRD-CIRAD, Collection à travers champs, 310 p.

7. Brunet, D., (1994). Un aménagement hydraulique simple pour la réhabilitation des sols salés: la riziculture en Basse Casamance. Sécheresse, 5, pp. 37-44.

8. Dent, D. (1986). Acid sulphate soils: a baseline for research and development. ILRI pub. N. 39, 204 p.

9. Descroix, L., Diongue, N. A., Panthou, G., Bodian, A., Sané, Y., Dacosta, H., Malam Abdou, M., Vandervaere, J-P., \& Quantin, G. (2015). Evolution récente de la pluviométrie en Afrique de l'ouest à travers deux régions: la Sénégambie et le bassin du Niger moyen. Climatologie, vol. 12, pp. 25-43. 
10. Diagne, M.T., \& Cescas M.P. (1997). Les méthodes d'analyses multidimensionnelles pour l'évaluation des sols de Basse-Casamance (Sénégal). Cahiers agricultures, 6, pp. 45-53

11. Diallo, N.H. (2016). Improved management of acid sulfate soils for rice production in Casamance, Senegal. (Master of Science), Virginia Polytechnic Institute and State University; 72 p + Annexes

12. Dresch, J. (1949). La riziculture en Afrique occidentale. Annales de géographie, 312 (53), pp. 295-312.

13. Fall, A.C.A.L., Montoroi, J-P., \& Stahr, K. (2014). Coastal acid sulfate soils in the Saloum River basin, Senegal. Csiro Publishing, Soil Research, 52, pp. 671-684.

14. Institut National de Pédologie (INP) \& Association pour la Lutte contre l'Exode Rural (Assolucer). (2009). Essais du Phosphogypse dans les rizières de Médina Souané. Délégation INP/ Fouladou-Pakao.

15. Khouma, M., \& Touré, M. (1982). Effects of lime and phosphorus on the growth and yield of rice in acid sulphate soils of the Casamance (Sénégal). In: Dost et al. (eds.): Proceedings of the Bangkok Symposium on acid su1phate soils. ILRI Pub. No. 31, pp. 237-250.

16. Khouma, M. (2006). Aperçu sur la toxicité ferreuse dans les Sols de rizières de Casamance. In: Audebert A. et al. Toxicité ferreuse dans les systèmes à base riz d'Afrique de l'ouest. Rapport ADRAO (Centre du Riz pour l'Afrique), pp. 146-156.

17. Linares, O.F. (1992). Power, prayer and production. The jola of Casamance, Senegal. Cambridge, Royaume-Uni, Cambridge University Press, $262 \mathrm{p}$.

18. Loyer, J-Y., Boivin, P., Le Brusq, J.-Y. \& Zante, P. (1988). Les sols du domaine fluvio-marin de Casamance (Sénégal): Evolution récente et réévaluation des contraintes majeures pour leur mise en valeur. Selected Papers of the Dakar Symposium on Acid Sulphate Soils (January 1986), H. Dost (ed.), ILRI, (pub.), Wageningen, The Netherlands, $\mathrm{N}^{\circ}$ 44, pp. 16-23.

19. Loyer, J-Y., Boivin, P., Le Brusq, J-Y., \& Zante P. (1986). Les sols du domaine fluvio-marin de Casamance (Sénégal) : évolution récente et réévaluation des contraintes majeures pour leur mise en valeur. Dakar: ORSTOM, 11 p. multigr.

20. Marius, C. (1985). Mangroves du Sénégal et de la Gambie: écologie, pédologie, géochimie, mise en valeur et aménagement. Paris, ORSTOM; 357 p. (Travaux et Documents de l'ORSTOM; 193). ISSN0371-6023 (Thèse Sciences Naturelles), Université Louis Pasteur, Strasbourg.

21. Montoroi, J-P. (1993). Les sols et l'agriculture dans le domaine estuarien de Basse Casamance. In Conservation et utilisation durable 
des ressources naturelles du bassin hydrographique de la Casamance (pp. 52-59), G. GREPIN, C. POMERLEAU, J.-Y. PIROT (éditeurs). AJAC-ZG, ISRA, ORSTOM, UICN, Ziguinchor-Dakar, Senegal.

22. Montoroi, J-P., Dobos, A., Fall, M., \& Sall, S. (1993). La réhabilitation de la riziculture inondée en Basse Casamance. ORSTOM Actualités, (40), pp. 2-7.

23. Montoroi, J-P., (1996a). Mise en valeur des bas-fonds en Basse Casamance (Sénégal). Agriculture et développement, 10, 61-74.

24. Montoroi, J-P. (1996b). Gestion durable des sols de la mangrove au Sénégal en période de sécheresse: dynamique de l'eau et géochimie des sels d'un bassin versant aménagé. Paris: ORSTOM, 1996, 263 p. (Etudes et Thèses). Thèse en Sciences de la Terre, Nancy 1.

25. Montoroi, J-P., \& Fall, A.C.A.L. (2017). Face à la salinisation des terres cultivées: la Casamance entre adaptation et diversification. In: Sciences au Sud - Le journal de l'IRD - n 84 - novembre 2016 à mars 2017.

26. N'gom, S. (1999). Etude de la salinité récurrente des sols dans le village de M'bassis et proposition de méthodes d'aménagements adaptées. (Mémoire de fin d'études), ENSA, 56 p.

27. Olivry, J.C. \& Chouret, A. (1981). Etude hydrologique du marigot de Bignona, Quelques aspects intéressants des mesures réalisées en 19701971. ORSTOM, Dakar, 1981, 93 p.

28. Pélissier, P. (1966). Les paysans du Sénégal. Les civilisations agraires du Cayor à la Casamance. Saint-Yriex, Imp. Fabrègue, 939 p.

29. Portères, R. (1950). Vieilles agricultures de l'Afrique intertropicale. Centres d'origine et de diversification variétale primaire et berceaux d'agricultures antérieurs au $\mathrm{XV}^{\mathrm{e}}$ siècle; Agronomie Tropicale, 9-10, pp. 489-507.

30. Posner, J.L., Kamuanga, M., \& Sall, S. (1988). Les Systèmes de production en Basse Casamance et les stratégies paysannes face au déficit pluviométrique. h4SU International Development Papers, Department of Agricultural Economics Agriculture Hall, Michigan S t a t e University, East Lansing, Michigan 48824-1039, U.S.A. Reprint No. 20F, USAID/ 47 p.

31. Sadio, S. (1991). Pédogenèse et potentialités forestières des sols sulfatés acides salés des tannes du Sine-Saloum (Sénégal), (Thèse de Doctorat) Université Agricole de Wageningen, Multigr/Trav. et Doc. Microf., ORSTOM, Paris, 270 p.

32. Sané, Y. (2016). Impacts de la dégradation des rizières par salinisation et ensablement dans la commune de Tenghory: de Tenghory Transgambienne à Diourou (Mémoire de Master). Université Assane Seck de Ziguinchor ; 102p. 
33. Vieillefon, J. (1977). Les sols des mangroves et des tannes de basse Casamance (Sénégal): importance du comportement géochimique du soufre dans leur pédogenèse. Mémoires ORSTOM 83; (Thèse Sciences Naturelles) Paris 6 : Paris, 298 p. ISBN 2-7099-0446-2

34. Vieillefon, J. (1975). Carte pédologique de Basse Casamance (domaine fluvio-marin) au 1/100 000, ORSTOM, 67p. 